\title{
Abstracts of the meeting of the Clinical Genetics Society and the Climical Molecular Genetics Society held on 30 and 31 March 1989 at the University of Southampton
}

\author{
A family with Turcot syndrome suggesting autosomal \\ dominant inheritance \\ D KUMAR*, C E BLANK*, AND B PONDER $\dagger$ \\ ${ }^{*}$ Centre for Human Genetics, Sheffield; and †Royal Cancer \\ Hospital, The Haddow Laboratories, Sutton, Surrey.
}

Turcot syndrome is a rare familial cancer syndrome with possible autosomal recessive inheritance. The essential features are neoplastic lesions in the brain (medulloblastoma, astrocytoma, glioblastoma) and colon (adenomatous polyps). Skin lesions (café au lait spots, benign pigmented naevi, and sebaceous cysts) are not uncommon. A family is presented with members from three generations who had a variety of skin tumours. The index patient had an astrocytoma excised from the right frontoparietal region and also had an adenomatous polyp removed from the descending colon. He also had a sebaceous carcinoma of the left lower eyelid. The clinical features are consistent with Turcot syndrome. The pedigree pattern here is suggestive of autosomal dominant inheritance. It is possible that Gardner's syndrome, familial polyposis coli, and Turcot syndrome are determined by the same allele. Studies using DNA probes mapping the 5q21-22 region, or allele specific probes, might elucidate this suggestion.

Identification of functioning sweat pores and visualisation of skin temperature patterns in females heterozygous for $X$ linked hypohidrotic ectodermal dysplasia and affected males by whole body thermography

K MACDERMOT, R P CLARK, AND M R GOFF

Clinical Research Centre, Watford Road, Harrow HAl $3 U J$.

Non-invasive thermography has been used for visualisation of individual sweat pores (SPRITE infrared) and skin temperature patterns (AGEMA 780 Thermovision) in subjects with XHED and in normal controls. Abnormal sweating patterns were seen in seven out of eight obligate carriers, all four affected males, and one out of six at risk females. The abnormalities consisted of horizontal asymmetrical areas with absent or reduced sweat pores visualised over the face and trunk. The thermal image of the back was modified to a 'cascade' of reducing temperatures not seen in six normal female controls. The distribution of sweating defects is compatible with the lines of Blaschko. Patchy (heterozygotes) or absent (males) 'wettedness' of the cornea and abnormal vasculature around the orbits were also seen. Confirmation of these findings by double blind study would facilitate XHED heterozygote detection in families with one affected male.
Adult polycystic kidney disease (APKD) in the north west: knowledge, experience, and attitudes to prenatal diagnosis in 100 kindreds

K A HODGKINSON, E A WATTERS, R ELLES, L KERZIN-STORRAR, AND R HARRIS

Department of Medical Genetics, St Mary's Hospital, Manchester M13 0JH.

One hundred and ninety subjects from 100 families with APKD on the NWRHA genetic register were interviewed in their homes between January 1987 and July 1988 to determine the likely demand for prenatal diagnosis, given the discovery of linkage to $\alpha$ globin on chromosome 16 . A detailed questionnaire was completed at interview to assess the understanding, and experience, of the clinical, therapeutic, and genetic aspects of APKD. A satisfactory understanding of each of these was found in $86 \%, 82 \%$, and $56 \%$ respectively. A total of $77 \%$ had thought that ultrasound was a reliable presymptomatic test and $75 \%$ felt that prenatal diagnosis should be available, of which onece third expressed interest. Unlike the remainder, most of these viewed the condition as serious, including nine who were either affected or at high risk and of reproductive age. However, only one request for prenatal diagnosis has so far been received. Demand for prenatal diagnosis appears to be very low and related to the perception of the seriousness of the disease.

Clinical application of predictive testing for Huntington's chorea using linked DNA markers

D CRAUFURD, L KERZIN-STORRAR, A DODGE, AND R HARRIS Departments of Psychiatry and Medical Genetics, University of Manchester.

Predictive testing using linked DNA markers is now offered to adults at risk for Huntington's chorea through our genetic register. Of 145 contacted so far, $54(37 \cdot 2 \%)$ accepted the offer of an appointment, and after preliminary counselling only $17(15.5 \%)$ are continuing. This contrasts with estimates of 50 to $80 \%$ uptake in previous surveys. We have now counselled 109 subjects (55 referred and 54 approached through the register), of whom $47(43.1 \%)$ are proceeding with a test, while 49 have declined or are undecided. Subjects proceeding with a test have been predominantly female (29/47), married (34/47), and already have children (29/47). Reasons given for requesting a test include resolution of uncertainty and clarification of risk to existing children, but surprisingly few (15/47) intend to base reproductive decisions on the test result and few requests for prenatal tests have been received. To 
date, 29 predictive tests have been completed, all but three informative. No significant differences were detected between gene carriers (12) and non-carriers (14) in the results of neurological and psychological examinations carried out before testing. No major adverse consequences have yet been observed.

Distinguishable transcripts of the putative testis determining sequences share homology with loci on chromosomes $\mathbf{X}$, $\mathbf{Y}$, and 9

N A AFFARA, D CHAMBERS, J O'BRIEN, S S M HABEEbU, M KALAITSIDAKI, C E BISHOP, AND M A FERGUSON-SMITH University of Cambridge Department of Pathology, Tennis Court Road, Cambridge CE2 IQP.

Oligonucleotide sequences based on the amino acid sequence of the putative testis determining gene ZFY have been used to isolate a $1.3 \mathrm{~kb}$ genomic fragment CMPXY1 from a $Y$ chromosome library and three cDNA reverse transcripts (CMPXY2, CMPXY3, and CMPXY4) from an adult human testis $\mathrm{cDNA}$ library. These sequences detect four potential exons on the $Y$, four on the $X$, and three of autosomal origin. The $\mathrm{X}$ linked sequences map to Xp21.2-Xp22.1 and studies with XX males locate the $Y$ linked sequences in distal Yp adjacent to the Y-autosomal homologous sequence GMGY3. These localisations have been confirmed by in situ hybridisation with CMPXY4 and have shown additionally that the autosomal sequences of CMPXY4 map to 9p22-9pter. Monosomy of 9p22-9pter is associated with anomalous sex differentiation in $\mathrm{XY}$ subjects. Restriction and sequence analysis indicates that CMPXY1/XY2/XY3 differ in sequence from CMPXY4, suggesting they are transcribed from different but closely related genes and that CMPXY4 must be either X linked or autosomal in origin. Thus more than one of the loci containing putative testis determining sequences are transcribed. This is consistent with northern blot analysis of RNA from human fetal testis where three low abundance transcripts of 5,6 , and $8 \mathrm{~kb}$ can be detected by the CMPXY3 cDNA sequence.

Use of dystrophin cDNA probes for differential diagnosis between Becker muscular dystrophy and limb-girdle muscular dystrophy

ANDREW NORMAN*, NICK THOMAS*, JOHN COAKLEY†, AND PETER HARPER*

*Institute of Medical Genetics, University of Wales College of Medicine, Cardiff; and tDepartment of Medicine, University of Liverpool.

The similarity in clinical features of $\mathrm{X}$ linked Becker muscular dystrophy (BMD) and autosomal recessive limbgirdle type muscular dystrophy (LGD) leads to difficulty in deciding on the correct diagnosis in the isolated male case. DNA probes (cDNA) complementary to the Duchenne/ Becker muscular dystrophy gene product, dystrophin, currently detect molecular deletions in 60 to $70 \%$ of cases of Duchenne/Becker muscular dystrophy. We have screened 18 isolated male cases of BMD with cDNA probes for the entire dystrophin gene; in 13 males $(72 \%)$ deletions were detected. We have also screened 15 male cases of LGD and detected similar deletions in four cases $(27 \%)$, thus reclassifying them as BMD. All male patients with progressive muscular dystrophy of limb-girdle pattern should be routinely screened with these cDNA probes as a useful adjunct to their clinical diagnosis, as the results clearly have important implications for counselling of their families.

Application of the polymerase chain reaction to diagnosis of Duchenne and Becker muscular dystrophy

R G ROBERTS, C G COLE, K A HART, M BOBROW, AND D R BENTLEY

Paediatric Research Unit, Guy's Hospital, London.

We have used a system based on the polymerase chain reaction (PCR) to perform prenatal and carrier diagnosis of Duchenne and Becker muscular dystrophy (DMD/ BMD). Deletion screening is carried out using three pairs of primers, which in combination detect $40 \%$ of all deletions. RFLP analysis is performed with primers which flank the polymorphic sites of pERT87-15/BamHI, pERT87-15/XmnI, and pERT87-8/TaqI; together these RFLPs exhibit heterozygosity in approximately $70 \%$ of women. We have characterised a novel insertion polymorphism ('MP1P') within the 3' untranslated region of the dystrophin gene. It is readily analysed with PCR, and is found to be heterozygous in about $25 \%$ of women. Out of 21 informative meioses, MP1P recombined with DMD twice (cf $\mathrm{C} 710 \%$ recombination). In one meiosis, recombination had occurred between MP1P and 99-6. MP1P, as a 3' flanking marker, represents a useful addition to our collection of intragenic PCR probes.

Linkage analysis in tuberous sclerosis: evidence for genetic heterogeneity with one disease locus at distal 9q J R SAMPSON*, J R W YATES $\dagger$, L A PIRRIT*, S POVEY\|, M W BURLEY\|, P FLEURY $\ddagger$, I WINSHIP§, P BEIGHTON§, A E FRYER ${ }^{* *}$, J OSBORNE $\dagger \dagger$, L AL-GAZALI $\ddagger$, R F MUELLER $\ddagger \ddagger$, AND J M CONNOR*

University Department of Medical Genetics, Duncan Guthrie Institute, Glasgow; +Department of Pathology, University of Cambridge; $¥$ Department of Neurology, University of Amsterdam; §Department of Human Genetics, University of Cape Town; $\| M R C$ Biochemical Genetics Unit, University College London; **University of Wales College of Medicine, Cardiff; +†Royal United Hospital, Bath; $¥ ¥$ Department of Genetic Counselling, Leeds General Hospital.

Evidence of linkage between tuberous sclerosis (TSC) and the $\mathrm{ABO}$ blood group and Abelson oncogene (ABL) was first reported by groups in the UK. Others, however, were unable to support these findings and when the original UK studies were extended the cumulative lod scores for these markers fell. Recently the observation of an infant with TSC who was trisomic for 11q23.2-qter led to speculation about a TSC locus in this chromosomal region. To clarify the situation eight rigorously assessed families affected by 
TSC were studied with nine markers: six from distal $9 q$ and three from distal 11q. The data as a whole supported a disease locus on distal $9 q$ (Zmax on LINKMAP analysis of the chromosome 9 probes being $3.776 \mathrm{cM}$ proximal to ABL). However, the programs HOMOG and HOMOG 2 indicated that TSC was likely to be genetically heterogeneous $(p=0.0112)$. In one family linkage to $\mathrm{ABL}$ was highly unlikely, while three others showed tight linkage. Reanalysis of the chromosome 9 data after exclusion of the unlinked family gave a multipoint lod score of $6 \cdot 1$ coincident with $\mathrm{ABL}$. Findings in the family not linked to ABL were consistent with a second disease locus on distal $11 \mathrm{q}$, but the family was too small to generate significant lod scores independently. Three further families investigated in the UK have proved informative for $A B L$ and on pooling our data evidence for heterogeneity has remained significant $(p=0 \cdot 02)$. Genetic heterogeneity in TSC will complicate efforts to clone the causative genes and limit the use of probes in clinical practice.

Linkage studies on an $\mathrm{X}$ linked centronuclear myopathy (CNM) family

JULIA STARR*, DAVID ROBINSON*, JOHN HARVEY*, AND MARGARET LAMONT $\dagger$

*Wessex Regional Cytogenetics Unit, Salisbury; and tDepartment of Child Health, Southampton General Hospital.

A previously unreported, three generation, $\mathrm{X}$ linked CNM family, in which the affected males all died shortly after birth, was studied to follow the inheritance of various $X$ chromosomal DNA probes with a view to determining the status of potential carrier females. The pedigree contained a number of obligate carrier females with multiple affected pregnancies. A previous study of two families suggested linkage of this condition to the Xq28 DNA markers ST14 (DXS52), DX13 (DXS15), and F8C. Our preliminary investigations using these and additional $\mathrm{Xq} 28$ probes $\mathrm{U} 6.2$ (DX304) and DX55-7 (DXS105) provide further evidence for the localisation of $\mathrm{CNM}$ to the $\mathrm{Xq} 28$ region.

Turner's syndrome: the use of DNA probes

DAVID ROBINSON*, PATRICIA JACOBS*, AND TERRY HASSOLD $\dagger$

*Wessex Regional Cytogenetics Unit, General Hospital, Salisbury, Wilts; and +Division of Medical Genetics, Emory University School of Medicine, Atlanta, Georgia 30322, USA.

The parental origin of the single $\mathrm{X}$ chromosome in sex chromosome monosomy can be determined by comparing restriction fragment length polymorphisms of $45, \mathrm{X}$ subjects or conceptuses with those of their parents. Such studies show that the single $\mathrm{X}$ can be derived from either parent but is usually maternal in origin. Approximately $50 \%$ of females presenting with Turner's syndrome have a cytogenetically detectable second cell line; however, it is possible that many more are 'cryptic' mosaics. We are currently using $X$ and $Y$ linked RFLPs to determine (a) the parental origin of the single $\mathrm{X}$ in subjects or conceptuses with a $45, X$ cell line; (b) whether the parental origin of the $\mathrm{X}$ affects the phenotype; and (c) whether we can detect 'cryptic' mosaicism in patients who have an apparently non-mosaic 45,X karyotype. Eight of 28 spontaneous abortions but only two of 17 liveborn Turner's syndrome have a paternally derived $\mathrm{X}$ chromosome. Furthermore, the two liveborn $X^{P}$ patients were atypical. These data suggest that the $\mathrm{X}^{\mathrm{P}}$ genotype may be less compatible with live birth than the $X^{M}$ genotype. We have tested $1145, X^{M}$ patients with Y linked probes to detect 'cryptic' mosaicism. No $Y$ chromosome material was detected in 10 patients, but one patient was positive for a Y material, although the band was of an unusual size, suggesting a possible $Y$ chromosome structural abnormality. Y chromosome mosaicism is of clinical importance if the second cell line contains $\mathrm{Y}$ chromosome material, as such patients are at risk of developing gonadoblastoma.

Gene probes in the diagnosis of familial hypercholesterolaemia

S HUMPHRIES*, R TAYLOR*, A DUNNING*, AND M SEED ${ }^{*}$

${ }^{*}$ Charing Cross Sunley Research Centre, London W6 $8 \mathrm{LW}$; and +Department of Medicine, Charing Cross and Westminster Medical School, Charing Cross Hospital, London W6 $8 R F$.

Familial hypercholesterolaemia (FH) is caused by a defect in the low density lipoprotein (LDL) receptor. It is a common disorder (heterozygote frequency 1 in 500) and 5 to $10 \%$ of men who have suffered a heart attack under the age of 55 years have FH. Diagnosis of FH in children would be useful, and for roughly $10 \%$ of cases where cholesterol levels do not allow an unambiguous diagnosis, DNA tests will be applicable. For the rare case of a fetus at risk of homozygous $\mathrm{FH}$, prenatal diagnosis will also be useful. In the UK, about $5 \%$ of patients with FH have a detectable deletion or rearrangement of part of the LDL receptor gene. In the families of the rest of patients, diagnosis may be possible using RFLPs detected with the LDL receptor probe. Using RFLPs detected with $N$ coI and ApaLI roughly $80 \%$ of patients are heterozygous for at least one of these RFLPS and this increases to over $85 \%$ using PvuII and StuI. Although the affected relatives of patients can be identified by these linkage methods, population screening is not feasible. Our current evidence from determining RFLP haplotype frequency suggests that there may be common mutations causing $F H$ in the UK. If such mutations could be identified, screening could be carried out using PCR and oligomelting.

The use of haplotype analysis in Scottish cystic fibrosis families

A CURTIS, M KESTON, S HOLLOWAY, AND D J H BROCK

Human Genetics Unit, University of Edinburgh, Western General Hospital, Edinburgh EH4 $2 \mathrm{XU}$.

DNA markers detect polymorphisms which show linkage disequilibrium with the mutant $C F$ locus to varying degrees in different populations and ethnic groups. In a study involving 150 patients and their families we have shown, using two of these diallelic markers, KM19 and XV2c, that linkage disequilibrium is particularly high in the Scottish 
population. The haplotype $\mathrm{K} 2 \mathrm{X} 1$, which is present in only $15 \%$ of normal chromosomes, is present in $89 \%$ of $\mathrm{CF}$ chromosomes. DNA typing, therefore, has allowed the CF heterozygote risk for a member of the population to be altered from a prior $5 \%$ to less than $1 \%$ or up to $24 \%$ and has been useful when counselling the spouses of close relatives of $\mathrm{CF}$ patients and in identifying 'low risk' $\mathrm{AI}$ donors. The linkage disequilibrium has also been applied in genetic counselling to increase the accuracy of risk probabilities in several prenatal diagnoses, which are more complex than an informative 1 in 4 risk pregnancy, for example, when DNA was unavailable from the index child. Risks can be further defined by combining haplotype and microvillar enzyme test results. Linkage disequilibrium and haplotype frequencies have provided estimates of the probability of a subject being affected with CF. Unless there is at least one $\mathrm{K} 2$ and one $\mathrm{X} 1$ allele, the probability is less than $1 \%$. These estimates, when combined with clinical considerations, have been important in the confirmation of diagnosis in some patients.

\section{Recombination or heterogeneity? Observations in some Manchester APKD families}

$R$ G ELLES, K HODGKINSON, A P READ, A WATTERS, AND R HARRIS

Department of Medical Genetics, St Mary's Hospital, Manchester.

Among 30 APKD families typed for markers flanking the PKD1 locus, we have found two pedigrees showing unusual numbers of recombinations. However, we do not think that these families are strong candidates for evidence of segregation of the PKD2 locus. If this second locus is present in an APKD family we would expect that approximately $50 \%$ of affected subjects would be apparently doubly recombinant for flanking chromosome 16p markers. In fact only one doubly recombinant subject is present in these families and one possible explanation of this observation is the occurrence of homozygosity at the PKD1 locus. We compare the two families published previously to show evidence of genetic heterogeneity with the pedigree we have observed, and calculate the likelihood that the patterns of marker inheritance they show are not likely to be the result of the operation of a second disease locus.

Alzheimer's disease locus maps close to the centromere on the long arm of chromosome 21

A M GOATE, A HAYNES, M J OWEN, L JAMES, M FARRALl, M J MULLAN, P ROQUES, M N ROSSOR, R WILLIAMSON, AND J A HARDY

Alzheimer's Disease Research Group, Departments of Biochemistry and Neurology, St Mary's Hospital Medical School, London W2 IPG.

Alzheimer's disease is the most common cause of dementia and a major cause of death in developed countries. Although the aetiology of the disease is unknown, epidemiological studies have consistently shown that a family history of $\mathrm{AD}$ is a major risk factor. In addition, many pedigrees have been reported in which the disease has an early onset $(<65$ years) and appears to segregate as an autosomal dominant with full penetrance. We and others have shown a genetic linkage between $\mathrm{AD}$ and DNA markers on chromosome 21 in 10 such families. Our results place the locus predisposing to familial AD on the long arm of chromosome 21 close to the centromere. This places the $\mathrm{AD}$ locus a considerable genetic distance proximal to the original linked marker (D21S1/S11). The two studies claiming to have excluded linkage to these markers will be discussed in the light of our recent results.

\section{Carrier and prenatal diagnosis by direct identification in haemophilia B \\ P M GREEN*, D R BENTLEY*, R S MIBASHAN $\dagger$, I M NILSSON $\ddagger$, AND F GIANNELLI* \\ ${ }^{*}$ Paediatric Research Unit, Guy's Hospital, +Department of Haematology, King's College Hospital; and $\ddagger$ Department of Coagulation Disorders, Malmo University Hospital, Sweden.}

Direct sequencing of genomic DNA amplified by the polymerase chain reaction (PCR) has been applied to the coagulation factor IX gene. The promoter, exons with flanking intron sequences, and the polyadenylation region of the $3^{\prime}$ non-coding tail of the factor IX transcript are amplified and sequenced to identify the detrimental mutation in 15 patients with the various clinical forms of the disease $\left(\mathrm{crm}^{+}, \mathrm{crm}^{\mathrm{R}}, \mathrm{crm}-\right.$, and inhibitors). This same approach is also used to detect mutations in women at risk of carrying the disease and in fetal samples. Single base substitutions and small deletions from one to eight base pairs are identified in hemizygous and heterozygous subjects. As analysis of all the amplified regions of the gene can be accomplished in three days, this procedure provides a practical diagnostic strategy that overcomes the difficulties of indirect DNA diagnoses.

\section{Maternal DNA in chorionic villus samples \\ J M OLD, K E HAN, AND D J WEATHERALL \\ National Haemoglobinopathy Reference Service, John Radcliffe Hospital, Oxford.}

Two of seven diagnostic errors in our prenatal diagnosis programme for the haemoglobinopathies were shown to be caused by maternal DNA contamination by hybridisation of a single locus hypervariable DNA probe. To determine the extent of contamination, $200 \mathrm{CVS}$ DNAs selected at random from our programme were analysed with the $\alpha$ globin $3^{\prime} \mathrm{HVR}$ and $\mathrm{p} \lambda \mathrm{g} 3$ probes. The two misdiagnosed CVS DNAs were found to be entirely maternal DNA. No paternal bands were present. Of the 200 random samples, 141 were positively identified to be maternal DNA free and 11 could be clearly identified as being contaminated $(7 \%)$. In one case the DNA was all maternal; in the other 10 the contamination was only slight (estimated at 1 to $5 \%$ ). Two cases of non-paternity were also identified. To determine if slight maternal DNA contamination would have affected a diagnosis by PCR, one of the 10 samples was amplified for 30 cycles. No evidence of amplification of the maternal DNA sequence was observed. 
The characterisation of three $\alpha_{1}$ antitrypsin deficient variants: Pi null Cardirf $-\mathrm{Asp}^{256} \rightarrow$ Val, $\mathrm{Pi}^{\text {Mmalton }}-\mathrm{Phe}^{51 / 52} \rightarrow$ deletion, Pil-Arg ${ }^{39} \rightarrow$ Cys

N KALSHEKER*, A GRAHAM, C R NEWTON, F BAMFORTH, $S$ J POWELL, AND A F MARKHAM

ICI Diagnostics, Gadbrook Park, Northwich, Cheshire CW9 7RA; and *Department of Medical Biochemistry, University of Wales College of Medicine, Cardiff CF2 1SZ.

Alpha $_{1}$ antitrypsin deficiency (AATD) is associated with predisposition to liver disease in childhood and lung disease in early adult life. We have characterised the mutations in three forms of AATD in heterozygotes by gene amplification and direct sequencing. In one family two subjects with $\mathrm{Pi}$ (proteinase inhibitor) Znull presented with recurrent respiratory tract infections in early childhood. In the null gene the codon for aspartate at position 256 has mutated to valine. In $\mathrm{Pi}^{\text {Mmalton }}$ there is a deletion of a codon either at position 51 or 52 and in PiI a single base substitution results in an arginine being replaced by cysteine at position 39 . These mutations occur in residues of the molecule which significantly affect tertiary structure, resulting in folding abnormalities which in all probability account for the deficiency states.

A study of $\mathrm{X}$ chromosome abnormality in XX males using dual laser, bivariate flow karyotype analysis and flow sorted dot blots

N P CARTER*, M E FERGUSON-SMITH $\dagger$, AND M A FERGUSONSMITH $\dagger$

*Department of Pathology, University of Cambridge; and †East Anglian Regional Genetic Service, Cambridge.

Dual laser flow cytometry produces bivariate flow karyotypes in which $\mathrm{X}$ chromosomes are clearly resolved from all others, improving the ability to detect small changes in DNA content. Chromosome preparations are stained with two fluorescent dyes, each with a different base pair specificity. Each chromosome passes, in flow, through two spatially separated lasers, the first laser exciting one dye, the second laser exciting the other. The independent measurements of the fluorescence intensity of both dyes is then correlated for each chromosome and displayed as a bivariate dot plot. The chromosomes are resolved not only by their DNA content but also by their base composition. Using this technique, we have studied chromosomes from lymphoblastoid cell lines established from $11 \mathrm{XX}$ males and have estimated the DNA content of both the normal and abnormal $\mathrm{X}$ chromosomes. The normal and abnormal $\mathrm{X}$ chromosomes from selected lines have also been flow sorted onto nitrocellulose filter paper and probed for the presence of $\mathrm{Y}$ sequences.

Early amniocentesis: experience of 222 cases

J NEVIN*, $N$ C NEVIN*, J C DORNAN†, D SIM†, AND M J ARMSTRONG $\ddagger$

${ }^{*}$ NI Regional Genetics Centre, Belfast City Hospital; tDepartment of Midwifery and Gynaecology, Queen's University of Belfast; and $¥$ Department of Midwifery and Gynaecology, Jubilee Maternity Hospital, Belfast.
Early amniocentesis is an alternative method for prenatal diagnosis. Since January 1987 , early genetic amniocentesis has been offered at the Regional Genetic Obstetric Clinic, Belfast, as an investigative procedure as early as 9.5 weeks after the last menstrual period. We report 222 amniocenteses performed before 15 weeks' gestation. Approximately $10 \mathrm{ml}$ of amniotic fluid was obtained following one insertion of a 20 gauge needle under continuous visualisation by ultrasound. Amniocytes were successfully cultured and harvested according to routine procedures. The average time to harvest was 10 days and the number and quality of metaphases were comparable to those from 16 week samples. Repeat amniocentesis was required in one case where the procedure had been performed at 12.0 weeks. There were seven abnormal karyotypes $(3 \cdot 2 \%)$, including four trisomy 21 , two trisomy 18 , and one with a metacentric chromosome. Two fetuses had spina bifida and Meckel's syndrome. No fetal loss has been observed to date and a careful follow up study is in progress.

Early amniocentesis: a cytogenetic evaluation of 60 cases M T REBELLO*, N MACLACHLAN $\dagger$, D E ROONEY*, J SMITH*, F E LOEFFLER ${ }^{*}$, R W BEARD* ${ }^{*}$ C RODECK $\dagger$, AND D V COLEMAN* *St Mary's Hospital, London W2; and TQueen Charlotte's Maternity Hospital, London W6.

Early amniocentesis represents a potential method of prenatal diagnosis which may avoid some of the disadvantages associated with chorionic villus sampling. We have undertaken a pilot study of 60 cases taken from pregnancies of seven to 14 weeks' gestation to evaluate the technique for cytogenetic analysis. Amniotic fluid samples were taken from 24 pregnancies of 12 to 14 weeks' gestation, and a result was obtained in all cases (success rate $100 \%$ ); 32 samples taken from pregnancies of seven to 11 weeks' gestation yielded a result in $66 \%$ of cases. On the basis of these results, early amniocentesis from 12 weeks' gestation is now being offered as a routine diagnostic procedure at St Mary's Hospital. Five cases received to date have all been successful. We are currently investigating culture methods to improve the success rate of samples taken before 12 weeks, in addition to the application of recombinant DNA techniques.

Maternal serum chorionic gonadotrophin levels in Down's syndrome pregnancies

J A CROSSLEY, D A AITKEN, AND J M CONNOR

Duncan Guthrie Institute of Medical Genetics, Yorkhill, Glasgow.

Human chorionic gonadotrophin (HCG) levels were measured in stored mid-trimester serum samples from 48 pregnancies affected by Down's syndrome, and compared with the levels found in 365 control samples from unaffected singleton pregnancies, matched for maternal age, completed weeks of gestation, and time in frozen storage. Alphafetoprotein (AFP) levels had been previously measured in these samples as part of the west of Scotland AFP screening service. The median HCG value of the pregnancies affected by Down's syndrome was found to be 
raised, at 2.3 multiples of the median (MOM) of unaffected pregnancies. Twenty-seven out of the 48 Down's samples $(58 \%)$ had HCG levels greater than 2.0 MOM, compared with 31 out of 365 control samples (8.5\%). Maternal serum HCG is a better discriminator of Down's syndrome pregnancies than maternal serum AFP. Combining HCG and AFP levels in MOM, as a ratio, further increases the discrimination, with $65 \%$ of Down's samples having a ratio greater than 2.3 compared with $8.5 \%$ of controls. Combining risks derived from the HCG(MOM)/AFP(MOM) ratios with mid-trimester maternal age risks for Down's syndrome, and using a cut off risk of 1:280, increases the potential overall detection rate to $67 \%$ and reduces the false positive rate to $6 \cdot 7 \%$. Using this method also has the advantage of reducing the potential amniocentesis rate in women under 30 and of increasing the detection rate in older mothers.

A dominantly inherited syndrome of microcephaly, sparse hair, mental retardation, and seizures

J A HURST, A VAN HAERINGEN, AND M BARAITSER

Institute of Child Health, 30 Guilford Street, London.

A Pakistani father and three of his seven children have microcephaly, sparse hair, and mild to moderate mental retardation. Two of the sons also have generalised seizures. One daughter has sparse hair only. The association of alopecia or sparse hair and seizures is recognised. This family most closely resembles those described by Moynahan and Shokeir. EEGs on our two boys have been compared to Moynahan's patient and do not show the same abnormalities. The family reported by Shokeir did not have microcephaly. The parents are distantly related but we consider that this family shows a new dominantly inherited microcephaly syndrome.

Parental origin in partial monosomy 4p (Wolf-Hirschhorn syndrome) using cytogenetics and DNA probes

HOWARD N HUGHES*, HELEN DALY $\dagger$, AND G T LEALMAN $\ddagger$ *Centre for Human Genetics, Sheffield; +Yorkshire Regional Cytogenetics Unit, Leeds; and $\ddagger$ Bradford Royal Infirmary, Bradford.

DNA probes defining the genetic loci D4S10,D4S43, and D4S95 were used to determine the paternal origin of a de novo partial monosomy $4 p$ when cytogenetic investigations showed both parents to have normal karyotypes.

\section{Noonan's syndrome: results of patient questionnaire M A PATTON AND $S$ BROWN \\ St George's Hospital, London SW17; and Noonan Syn- drome Society.}

Patient questionnaires for 53 subjects with Noonan's syndrome (mean age $\mathbf{9 . 8}$ years) were analysed. Neonatal oedema was present in $28 \%$, while $50 \%$ required neonatal care, usually for feeding difficulties. Cardiac involvement was present in $88 \%$ : pulmonary stenosis $43 \%$, ASD $23 \%$, and cardiomyopathy $15 \%$. A relatively high proportion of visual problems other than ptosis was found $(60 \%)$. Of the 29 of school age, 20 were receiving special education. There was recurrence in only three families. Patient group questionnaires have a biased ascertainment, but often draw attention to patients' concerns.

Ivemark's syndrome and Müllerian duct anomaly in monozygotic twins

J C S DEAN*, E S GRAY $\dagger$, N E HAITES*, D J LLOYD $\dagger$, K ROSS*, AND J WEIR§

Departments of Medical Genetics*, Pathologyt, and Child Health $\ddagger$, University of Aberdeen; and \$Department of Radiology, Aberdeen Royal Infirmary.

Ivemark's syndrome, the association of congenital heart disease with asplenia or polysplenia, is often accompanied by other features of disordered lateralisation. Midline or symmetrical structures are usually normal. We report monozygotic female twins (zygosity confirmed by DNA fingerprinting) both of whom had bifid uterus and vagina with hypoplastic ovaries in addition to hypoplastic left heart syndrome, polysplenia, malrotation of the gut, and abnormal pulmonary septation. One twin had mildly dysmorphic facies and both had neck webbing, but karyotypes were normal female, 46,XX. Genital tract abnormality has not been reported previously with polysplenia in Ivemark's syndrome, although there is one case reported with asplenia. Failure of midline fusion of the Müllerian ducts could well be another feature of the developmental disturbance which results in the left and right isomerism of this laterality sequence.

Patent ductus arteriosus with characteristic facies: an autosomal dominant syndrome

HEATHER R DAVIDSON

Duncan Guthrie Institute of Medical Genetics, Glasgow G3 8SJ.

We describe a large family in which patent ductus arteriosus (PDA) and unusual facial appearance affects at least nine subjects and segregates as an autosomal dominant trait. The syndrome shows incomplete penetrance with regard to the PDA in that four adults who transmit the gene have no clinical signs or history of PDA although spontaneous closure of an asymptomatic duct cannot be excluded. These subjects do, however, show the same characteristic facial features as the other affected relatives, in particular a broad, high forehead and a short nose with broad, flattened tip. There have been several previous descriptions of families showing autosomal dominant transmission of PDA but no mention was made of their facial appearance.

Investigation of a 'balanced' translocation by flow cytometry J L TOLMIE, A COOKE, C GREIG, J COLGAN, AND J M CONNOR Duncan Guthrie Institute of Medical Genetics, Glasgow.

Cytogenetic analysis on peripheral blood lymphocytes from a child with impaired intelligence, minor dysmor- 
phism, obesity, and genital hypoplasia showed an apparently balanced translocation, $46, \mathrm{XY}, \mathrm{t}(4 ; 14)(\mathrm{q} 12 ; \mathrm{q} 13)$. The same chromosome rearrangement was detected in the child's father who had subtle phenotypic abnormalities similar to those present in his son. Detailed study of flow karyotypes produced from lymphoblastoid cell lines established that in both patients the translocation was in fact unbalanced with approximately 11 million base pairs of DNA (corresponding to around $6.0 \%$ of chromosome 4 or $11.0 \%$ of chromosome 14 ) being lost.

Frontonasal dysplasia and Poland's syndrome W REARDON, I K TEMPLE, J HURST, AND M BARAITSER Institute of Child Health, London.

Skeletal malformations in frontonasal dysplasia are rare. One case reported in 1950 documented the occurrence of pectoral muscle hypoplasia in a case of FND. We describe a 10 year old girl with classic features of FND (hypertelorism, broad nasal root, bifid nasal tip with ala nasi notching on the right side) who has a right sided Poland's anomaly with 3-4 skin syndactyly in the right hand. FND comprises a spectrum of abnormalities which may, occasionally, include Poland's anomaly.

Prenatal diagnosis of a double bisatellited marker: confirmation of diagnosis by flow sorting

S HODGSON, J J WATERS, M E FERGUSON-SMITH, N CARTER, J ALEXANDER, R NASH, AND J HAWKINS

Addenbrooke's Hospital, Hills Road, Cambridge CB2 $2 Q Q$.

Prenatal diagnosis, by amniocentesis, showed a bisatellited, dicentric, DADDAPI positive, de novo marker present in two copies in $20 \%$ of cells from more than one culture. After counselling the parents elected for termination of pregnancy. Placental and fetal tissue confirmed the presence of the markers at approximately the same level of mosaicism. A lymphoblastoid cell line established from cardiac blood was used to confirm the presence of the double marker by flow sorting. The fetus was normal at necropsy.

A case of partial monosomy of short arm of chromosome 8 with unbalanced rearrangement of an unknown satellited material

D KUMAR*, M CLARKE*, AND S A W SALFIELD $\dagger$

*Centre for Human Genetics, 117 Manchester Road, Sheffield; and +Department of Paediatrics, District General Hospital, Rotherham.

Cytogenetic investigations of a seven year old girl with dysmorphic features and mild mental retardation showed an unbalanced rearrangement of chromosome 8 . This had resulted in monosomy of the terminal region of $8 p$ and extra satellited material of unknown origin: $46, X X,-8,+\operatorname{der}(8)(8 q t e r-8 \mathrm{p} 23.1:: S$ ? $)$. The karyotypes of both parents were normal. Comparison of the phenotypic features of other reported cases of partial monosomy $8 p$ suggests there may be some similarities between these patients.

Prospective screening for autosomal trisomies in the west of Scotland using a combination of MSAFP and age, July 1987 to June 1988

D A AITKEN, J A CROSSLEY, AND J M CONNOR

Duncan Guthrie Institute of Medical Genetics, Yorkhill, Glasgow G3 8SJ.

Since July 1987 , prospective prenatal screening for autosomal aneuploidies, based on a combination of the risks derived from $\alpha$ fetoprotein (AFP) levels and maternal age, has been under way in the west of Scotland. In the first 12 months of this programme 30000 women were screened and a total of 956 pregnancies $(3 \cdot 2 \%)$ was identified which had a combined risk exceeding a threshold approximating to the age related risk of 35 years old at mid trimester. Eight chromosomally abnormal fetuses were identified within this high risk group and a further five abnormalities were identified in 360 women of advanced maternal age ( $\geqslant 35$ years) proceeding directly to amniocentesis who did not have serum AFP screening. An analysis of the available pregnancy outcome data indicates that selectivity has been enhanced to one abnormality per 100 pregnancies identified at high risk compared with 1:125 for age only screening. There was also a notable improvement in compliance after the introduction of the programme with a doubling of the amniocentesis rate in the 35 years and over age group identified at high risk.

Genetic analysis of different kinds of PKU in one family L A TYFIELD*, A L MEREDITH $\dagger$, $M$ J OSBORN*, AND P S HARPER $\dagger$

${ }^{*}$ Molecular Genetics Section, Department of Biochemistry, Southmead Hospital, Bristol; and HInstitute of Medical Genetics, University of Wales College of Medicine, Cardiff.

Phenylketonuria (PKU) is a heterogeneous disorder at the clinical and biochemical levels. An increased understanding of the molecular genetics of PKU is making it possible to relate differences in phenotypic expression to different haplotype patterns and specific mutations at the phenylalanine hydroxylase (PAH) gene locus. We report the results of biochemical and molecular analysis on an extended non-consanguineous family in which two children who are first cousins had different biochemical presentations of PKU in the neonatal period. The older child was treated for 'classical' PKU; the cousin, 15 years younger, has a milder form of the disorder but some degree of phenylalanine restriction is required. Using a full length cDNA probe for the PAH locus, we have shown that both children have inherited the same mutant allele from their grandfather who also has PKU which was previously undiagnosed (phenylalanine $>1000 \mu \mathrm{mol} / \mathrm{l}$ ). $\mathrm{He}$ is of normal intelligence and has never had dietary protein restriction. All affected family members have a different haplotype pattern on their second allele. We are attempting to correlate haplotype patterns with biochemical and clinical status. 
Estimating the probability of gonadal mosaicism in mothers of boys with Duchenne muscular dystrophy from maternal creatine kinase levels

I $K$ TEMPLE, $S$ HODGSON, A ADES, AND M E PEMBREY

Institute of Child Health, London.

Gonadal mosaicism occurs when an early somatic mutation involves a proportion of germ line cells but leaves most of the somatic cells intact. This has been recognised as a possibility for many years but has recently been highlighted in Duchenne muscular dystrophy (DMD) where deletion at the DMD locus can be detected in affected brothers, despite two non-deleted alleles at that locus in maternal white blood cell DNA. We propose a method for estimating the percentage of DMD 'carriers' who have gonadal mosaicism using $\mathrm{CK}$ data from two pooled sets (ICH and Hammersmith Hospital using the same method [SCE CK-NAC method]). We compared curves of log CK and frequency for three groups of patients: (1) normal women, (2) true obligate carriers with a positive history in two generations, and (3) obligate carriers (so called because they have two affected boys but no other affected relatives). Assuming curve 3 is a composite of curve 1 and 2 the best fit indicates that $19 \cdot 1 \%$ of group 3 come from a population of normal women with respect to their CK values and therefore may be gonadal mosaics. A multicentre study is required to obtain larger numbers and we would like to invite interested parties to consider such a collaboration.

\section{Physical mapping around the steroid sulphatase locus}

R S NEWMAN, N A AFFARA, E GILlARD, M MITCHELl, J R W YATES, A BALLABIO, AND M A FERGUSON-SMITH

Department of Pathology, University of Cambridge, Cambridge.

The steroid sulphatase locus has been localised to Xp22.3-pter. We have found that $80 \%$ of patients with $X$ linked ichthyosis (familial steroid sulphatase deficiency) are deleted completely for the STS locus and the nearby anonymous sequences GMGX9, GMGXY19, and GMGXY3. Flow cytometry indicates that in some cases approximately $3 \mathrm{Mb}$ of DNA has been lost. The high frequency of deletion suggests that a particular mechanism of mutation may be occurring, perhaps aberrant XY interchange with the STS homologous pseudogene on Yq. We are constructing a long range physical map around STS using pulse field electrophoresis to place GMGX9, GMGXY19, GMGXY3, and other probes relative to STS. Preliminary results show that GMGX9 and the $5^{\prime}$ end of the STS gene can be linked physically on a $800 \mathrm{~kb}$ SacII fragment. GMGXY3 detects a $850 \mathrm{~kb}$ SacII fragment distinct from that carrying GMGX9 and the 5' end of the STS gene. We are currently trying to link these fragments. This map will be used to examine and clone the deletion breakpoints in STS patients.

Localisation of three zinc finger genes using Southern analysis of somatic cell hybrid DNA and non-radioactive in situ hybridisation
S S M HABEEBU, J GIBSON, N A AFFARA, AND M A FERGUSONSMITH

Department of Pathology, University of Cambridge, Cambridge.

Three DNA sequences (CMPX1, 5CMP1, and 16.2) were isolated from a human testes cDNA library by screening with a probe containing a zinc finger motif. These sequences were shown to contain zinc finger domains by partial DNA sequencing. Southern blot analysis of genomic DNA from somatic cell hybrids containing deleted $\mathrm{X}$ chromosomes and various complements of autosomes mapped CMPX1 to Xq13-Xq24 and 5CMP1 to chromosome 5 . This panel of hybrids was not informative for $16 \cdot 2$. These localisations were confirmed and more precisely determined by in situ hybridisation using these probes labelled with biotin. The detection system used was avidin-peroxidase-DAB with reflection contrast microscopy. The analysis showed that CMPX1 mapped to the boundary between $\mathrm{Xq} 13$ and $\mathrm{Xq} 21$. Interestingly, 5CMP1 was shown by in situ hybridisation to map to two distinct loci on chromosome 5; one on 5p13-5p14 and the second on $5 q 12-5 q 13$. This result suggests that two genes exist on chromosome 5 that contain sequences closely related to 5CMP1. In situ analysis with $16 \cdot 2$ has yet to be completed. This study shows that our in situ hybridisation technique is sufficiently sensitive to detect and map accurately single copy gene sequences.

DMD/BMD carrier detection using a new RFLP ANDREW READ AND ROGER MOUNTFORD

Department of Medical Genetics, St Mary's Hospital, Manchester M13 OJH.

The dystrophin cDNA probe Cf56a (equivalent to Kunkel's probe 8 ) detects a high frequency BanI polymorphism. BanI filters give constant bands of $17,13,8 \cdot 5,6 \cdot 4$, and $1 \cdot 35$ $\mathrm{kb}$, and a polymorphic band with alleles of $6 \cdot 7,6 \cdot 5$, and 4 $\mathrm{kb}$. Allele frequencies are $0.66,0.22$, and 0.12 (50 chromosomes) giving a PIC of $0 \cdot 45$. The variable band corresponds to the $5 \cdot 1 \mathrm{~kb}$ Pst I band, exon $\mathrm{J}$ in Read et al (Hum Genet 1988;80:152-6). Deletion of this exon is one of the commonest causes of DMD or BMD; in our series of 170 unrelated patients $42(25 \%)$ had this exon deleted. Thus, this RFLP is particularly useful for modifying carrier risks by showing heterozygosity or apparent non-maternity in at risk females. We show examples of its use.

DNA 'fingerprint' changes in patients on combination chemotherapy

K ROSS*, N HAITES $\dagger$, K KELLY $\dagger$, A DAWSON*, AND B BENNETT* Departments of Medicine and Therapeutics* and Genetics and Microbiology + , University of Aberdeen, Aberdeen.

Hypervariable minisatellite DNA probes 33.6 and $33 \cdot 15$ have been used in a new way in a group of patients receiving combination chemotherapy in the treatment of lymphoma. The multilocus nature of these probes allows simultaneous observation of over 60 different sites in human genomic DNA, enhancing the possibility of 
observing change. In five patients out of 13 already studied, changes have been observed in their peripheral leucocyte derived DNA fingerprint at some point after starting treatment. Changes involve both the loss of bands present in pretreatment samples and the appearance of new bands. Control studies indicate that the changes observed are unlikely to be artefactual. Some changes appear to be sensitive to the presence of spermidine in the restriction digest, and we conclude that changes observed may represent site specific modification of the DNA by one or more of the agents used in chemotherapeutic regimens.

A study of seven myotonic dystrophy families JOHN R QUICK*, SIMON LONGSTAFF $\dagger$, AND DIANA CURTIS $\ddagger$ ${ }^{*}$ Centre for Human Genetics, Sheffield; tDepartment of Ophthalmology, Royal Hallamshire Hospital, Sheffield; and $¥$ Sub Department of Medical Genetics, University of Sheffield.

We report haplotypes in seven families with MD. We note a large degree of linkage disequilibrium between alleles around the Apo CII gene. This is in agreement with Wallis et al and Appleby et al. Comparison of carrier detection by DNA probe analysis and routine clinical examination (neurological and ophthalmological tests) is made.

Carrier detection and prenatal diagnosis in Norrie's disease D CURTIS*, C E BLANK* ${ }^{*}$ AND H N HUGHES $\dagger$

*Sub-Department of Medical Genetics, and +Centre for Human Genetics, 117 Manchester Road, Sheffield.

A family in which Norrie's disease (NDP) is segregating is described. NDP is segregating with allele I of the RFLP recognised by DNA probe $\mathrm{L} 1.28(\mathrm{Taq})$ at the $D X S 7$ locus. Three young women have been assessed for carrier status; one of these women has had two prenatal diagnoses using linkage to $\mathrm{L} 1.28$ as the diagnostic criteria. Her first pregnancy, a male at high risk for NDP, was terminated; her second pregnancy, a male at low risk for NDP, is continuing to term.

RFLP analysis of the Acyl-CoA dehydrogenase medium chain (ACADM) region

ALEX BLAKEMORE* ${ }^{*}$ PAUL C ENGEL ${ }^{*}$, AND DIANA CURTIS $\dagger$

${ }^{*}$ Department of Biochemistry, and +Sub-Department of Medical Genetics, Sheffield University.

Medium-chain acyl CoA dehydrogenase (ACADM) deficiency is an episodic disorder of $\beta$ oxidisation which has been implicated in cot deaths. It is inherited as an autosomal recessive condition (chromosome $1 \mathrm{p} 31$ ) and is notoriously difficult to diagnose as clinical symptoms are only present when the patient is in crisis. In this study, a full length cDNA clone of the human liver ACADM gene was used as a probe to screen the general population for diagnostically useful RFLPs. The poster describes the characterisation of three common RFLPs identified in this way.
Molecular analysis of the $X$ chromosome in Turner's syndrome

S A R LOUGHLIN, A REDHA, L A PIRRIT, E BOYD, AND J M CONNOR

Duncan Guthrie Institute of Medical Genetics, Glasgow.

Thirty-two nuclear families with a child or fetus with Turner's syndrome were studied using DNA restriction fragment length polymorphisms. A variety of karyotypes were represented in the sample including $45, X$, mosaic 45, $X / 46, X$ r $(X), 46, X$ iso $(X q)$, mosaic $45, X / 46, X X$, mosaic $45, X / 46, X$ iso(Xq), and $46, X X p-$. Using the DNA probe St14 (DXS52) which maps to Xq28 and four short arm probes (L1.28, XJ1.1, 87.15, and p19B) parental origin of non-disjunction was determined in 24 cases. We found loss of the paternal homologue to be much more frequent in this study. Analysis with both short and long arm probes allowed determination of the origin of isochromosome and ring formation. In the ring chromosome, linear order of previously mapped probes was preserved.

Physical and genetic studies of neurofibromatosis type 1 (von Recklinghausen NF)

D JADAYEL, C MATHEW, AND B A J PONDER

Section of Human Cancer Genetics, Institute of Cancer Research, Sutton, Surrey.

The von Recklinghausen neurofibromatosis (NF1) locus has been mapped to the proximal long arm of chromosome 17. We have screened a panel of 41 patients for deletions or rearrangements at this locus. This was carried out using the pulsed field gel electrophoresis technique (PFGET). DNA extracted from lymphocytes was digested with NotI enzyme and hybridised with two flanking but closely linked markers to NF1; HHH202/D17S33 $(Z=33.3$ at $\theta=0.01)$ and EW206/D17S57 $(Z=20.7$ at $\theta=0.03)$. In all the patients, as in control DNAs, HHH202 and EW206 probes hybridised to NotI fragments of 600 and $400 \mathrm{kbp}$ respectively, indicating absence of deletions or rearrangements in the close proximity of these markers. We are constructing restriction maps around these two probes by means of PFGET. This would provide necessary information to isolate new DNA markers several hundred kilobases closer to the NF1 gene. We are also attempting to establish whether NF1 mutations occur more frequently on chromosomes of maternal or paternal origin. Five three-generation families with new NF1 mutations are being typed with markers closely linked to NF1. The mutation was of paternal origin in the first family in which the analysis has been completed.

Clinical features and prognosis of von Hippel-Lindau disease

E $R$ MAHER, $R$ HARRIS, $J$ R W YATES, AND M A FERGUSONSMITH

Department of Pathology, University of Cambridge, and Radiology Department, Grimsby General Hospital.

Clinical features and survival of 76 previously unreported patients with von Hippel-Lindau disease were analysed. 
Seven were sporadic cases and 69 were familial cases from 29 kindreds. The most frequent complications (\% affected, mean age at diagnosis, and range of age at diagnosis respectively) were: cerebellar haemangioblastoma $(61 \%$, 28.4 years, 14 to 54 years), retinal angioma $(57 \%, 24.9$ years, seven to 46 years), renal carcinoma $(28 \%, 43.7$ years, 24 to 64 years), and spinal haemangioblastoma $(21 \%, 33.9$ years, 11 to 60 years). Phaeochromocytoma developed in nine patients from six families (mean age at diagnosis $=17.8$ years, range 12 to 28 years). Age at onset curves were constructed and protocols for screening at risk relatives and for following up affected patients are proposed. A Kaplan-Meier survival curve was constructed and the median actuarial survival from birth was 49 years.

Analysis of Scottish Duchenne and Becker muscular dystrophy families with cDNA probes

A COOKE, W G LANYON, E F GILlARD, E S DORNAN, D E
WILCOX, A McWHINNIE, A MORRIS, M A FERGUSON-SMITH, AND J M CONNOR

Duncan Guthrie Institute of Medical Genetics, Glasgow.

A total of 127 Scottish families with at least one subject affected by DMD (104) or BMD (23) was studied with the series of cDNA probes isolated by Koenig et al (1987), excluding the $3^{\prime}$ region of the gene (probes 10 to 14$)$. Using mainly HindIII digested DNA from affected males, 78 patients showed deletions of HindIII fragments (corresponding approximately to dystrophin exons), ranging from one to 32 fragments in size. A single patient was also detected with a duplication of three HindIII fragments. The deletions were found to be concentrated in the area of probe cDMD 8, with 51 patients failing to hybridise to at least one of the fragments detected by this probe. Endpoints of deletions were determined either precisely or with a maximum variability of two HindIII fragments in 70 patients while the remaining patients had a single deletion endpoint defined. No significant difference in deletion frequency was found between DMD (62 out of 104) and BMD (16 out of 23). 